\title{
Surgical Management of Intrahepatic Cholangiocarcinoma in Patients with Cirrhosis: Impact of Lymphadenectomy on Peri-Operative Outcomes
}

\author{
Fabio Bagante ${ }^{1} \cdot$ Gaya Spolverato $^{1} \cdot$ Matthew Weiss $^{2} \cdot$ Sorin Alexandrescu $^{3}$. \\ Hugo P. Marques ${ }^{4} \cdot$ Luca Aldrighetti $^{5}$ - Shishir K. Maithel ${ }^{6}$. Carlo Pulitano ${ }^{7}$. \\ Todd W. Bauer ${ }^{8}$ - Feng Shen ${ }^{9}$ George A. Poultsides ${ }^{10}$ - Olivier Soubrane ${ }^{11}$. \\ ${\text { Guillaume } \text { Martel }^{12} \text { • B. Groot Koerkamp }}^{13}$ • Alfredo Guglielmi ${ }^{1} \cdot$ Endo Itaru $^{14}$. \\ Andrea Ruzzenente $^{1} \cdot$ Timothy M. Pawlik ${ }^{15}$ \\ Published online: 3 January 2018 \\ (C) Société Internationale de Chirurgie 2018
}

\begin{abstract}
Background The consequences of lymphadenectomy (LND) on cirrhotic patients undergoing hepatectomy for intrahepatic cholangiocarcinoma (ICC) have not been investigated. We sought to analyze the impact of LND on morbidity among patients undergoing resection for ICC.

Methods A total of 1005 patients who underwent hepatectomy for ICC at one of the 14 participating institutions between 1990 and 2015 were identified. A propensity score match analysis was performed to reduce confounding biases between cirrhosis and non-cirrhosis groups.

Results Cirrhosis was diagnosed in 118 (11.7\%) patients. Among non-cirrhotic patients, 63\% underwent major liver resection versus only $20 \%$ among patients with cirrhosis $(p<0.001)$. LND was also less common among cirrhotic versus non-cirrhotic patients (19 vs. $50 \%, p<0.001)$. The incidence of complications was 41 and $30 \%$ among patients who did not and did have cirrhosis, respectively $(p=0.022)$. The propensity-matched cohort included 150 patients. The incidence of complications was $71 \%$ among patients who underwent lymphadenectomy versus $23 \%$ among patients who did not undergo lymphadenectomy (OR 8.39) $(p<0.001)$. In the propensity-matched analysis, the median HLN was comparable among patients independent of cirrhosis status (median HLN: non-cirrhosis, 2.5 vs. cirrhosis, 2) $(p=0.95)$. While lymphadenectomy was associated with a higher risk of infections (non-cirrhosis, $0 \%$ vs. cirrhosis, $21 \%, p<0.001$ ) among patients with cirrhosis, infections were not associated with lymphadenectomy among non-cirrhotic patients $(p=0.19)$.

Conclusion Lymphadenectomy was associated with an increased risk of complications among patients with cirrhosis undergoing surgery for ICC. The benefit of lymphadenectomy in cirrhotic patients should be considered in light of the higher risk of postoperative complications compared with non-cirrhotic patients.
\end{abstract}

Electronic supplementary material The online version of this article (https://doi.org/10.1007/s00268-017-4453-1) contains supplementary material, which is available to authorized users.

Fabio Bagante and Gaya Spolverato have contributed equally to this manuscript.

\section{Timothy M. Pawlik}

tim.pawlik@osumc.edu

Department of Surgery, University of Verona, Verona, Italy

2 Department of Surgery, Johns Hopkins Hospital, Baltimore, MD, USA

\section{Introduction}

A variety of liver diseases including intrahepatic lithiasis, primary sclerosing cholangitis, congenital abnormalities of the bile ducts, and liver fluke infection can induce a state of

3 Department of Surgery, Fundeni Clinical Institute, Bucharest, Romania

4 Department of Surgery, Curry Cabral Hospital, Lisbon, Portugal

5 Department of Surgery, Ospedale San Raffaele, Milan, Italy 
chronic biliary inflammation resulting in severe fibrosis and cirrhosis of the underlying liver parenchyma [1-3]. Several studies have identified cirrhosis as a risk factor for the development of intrahepatic cholangiocarcinoma (ICC) $[1,2,4-6]$. In fact, a strong association between HCVrelated cirrhosis and ICC $[7,8]$ and a weaker but consistent association between HBV-related cirrhosis and ICC have been identified [9]. Furthermore, cirrhosis associated with metabolic syndrome, steatohepatitis, and non-alcoholic fatty liver disease (NAFLD) has been linked to an increased risk of ICC $[10,11]$. A population-based analysis using the Surveillance, Epidemiology, and End Results (SEER) database confirmed a strong association between cirrhosis and ICC [12].

Recently, the 8th edition of the American Joint Committee on Cancer (AJCC) staging manual emphasized the importance of adequate nodal staging for ICC [13]. The manual called for a minimum of six harvested lymph nodes (HLN) for adequate nodal staging. The potential implications of performing lymphadenectomy among patients with ICC and cirrhosis have not been investigated. This topic is of particular importance because the subset of patients with cirrhosis may be at increased risk of morbidity [14]. For example, Lee et al. [15] reported that patients with cirrhosis who underwent gastrectomy and a D2 lymphadenectomy for gastric cancer had a higher incidence of postoperative complications of up to $40 \%$. In a separate study, Tachibana et al. [16] reported that the incidence of postoperative complication was as high as $80 \%$ among patients with cirrhosis who underwent esophagectomy combined with an extended lymphadenectomy.

6 Department of Surgery, Emory University, Atlanta, GA, USA

7 Department of Surgery, Royal Prince Alfred Hospital, University of Sydney, Sydney, Australia

8 Department of Surgery, University of Virginia, Charlottesville, VA, USA

9 Department of Surgery, Eastern Hepatobiliary Surgery Hospital, Shanghai, China

10 Department of Surgery, Stanford University, Stanford, CA, USA

11 Department of Hepatobiliopancreatic Surgery and Liver Transplantation, AP-HP, Beaujon Hospital, Clichy, France

12 Division of General Surgery, Department of Surgery, University of Ottawa, Ottawa, ON, Canada

13 Department of Surgery, Erasmus University Medical Centre, Rotterdam, Netherlands

14 Gastroenterological Surgery Division, Yokohama City University School of Medicine, Yokohama, Japan

15 Department of Surgery, The Urban Meyer III and Shelley Meyer Chair in Cancer Research, The Ohio State University Wexner Medical Center, 395 W. 12th Ave., Suite 670, Columbus, OH 43210, USA
While the impact of cirrhosis on morbidity related to hepatectomy has been well defined [17], the possible increase in morbidity related to lymphadenectomy among patients with ICC and cirrhosis has not been examined. Therefore, the objective of the current study was to define the impact of cirrhosis on lymphadenectomy utilization relative to short-term outcomes following surgery for ICC using a large multi-institutional international dataset.

\section{Patients and methods}

\section{Study population and data collection}

Patients who underwent hepatectomy for ICC at one of 14 hepatobiliary centers between 1990 and 2015 were identified (Supplementary Methods). Only patients who underwent curative-intent hepatectomy for ICC without extrahepatic metastasis were included. Patients who underwent only non-surgical treatments such as percutaneous ablation or intra-arterial therapy were excluded. Standard patient demographic and clinicopathological characteristics were collected including age, gender, American Society of Anesthesiologist (ASA) class, serum level of CA19-9, morphological type, number of ICC, tumor size, invasion of adjacent organs, liver capsule involvement, margin status, tumor grade, major vascular invasion, lymphovascular invasion, perineural invasion, and lymph-nodal status were collected. The diagnosis of cirrhosis was histologically confirmed. Treatment-related data such as type and extent of hepatectomy, lymphadenectomy, receipt of neoadjuvant, and adjuvant chemotherapy were also recorded. Tumor stage was categorized according to the 8th edition of the American Joint Committee on Cancer (AJCC) [13].

\section{Statistical analysis}

Continue variables were described as medians with interquartile range (IQR), and categorical variables were recorded as totals and frequencies. Univariable comparisons were assessed using Chi-squared test or Fisher's exact test as appropriate. To account for any potential residual confounders regarding the effect of cirrhosis on postoperative complications, propensity scores matching was performed (Supplementary Methods). The degrees of covariate imbalance in unmatched and matched samples were measured using the standardized differences as proposed by Austin et al. [18]. A $p$ value of $<0.10$ (two-tailed) was considered statistically significant. All analyses were performed using Stata version 12.0 (StataCorp LP, College Station, TX, USA) or R software for statistical computing, 
v. 3.0.2 34, with the additional packages: Hmisc and Matching.

\section{Results}

\section{Baseline characteristics of patients in non-cirrhosis and cirrhosis groups}

A total of 1005 patients underwent hepatectomy for ICC. Cirrhosis was present in $118(11.7 \%)$ patients, while 887 $(88.3 \%)$ patients did not have cirrhosis (Table 1). All patients who had cirrhosis were Child-Pugh Class A and well compensated. Several clinical characteristics were different between the cirrhotic versus non-cirrhotic groups (Supplementary Materials). At the time of surgery, patients who did not have cirrhosis $(n=494,63.2 \%)$ more often underwent a major hepatectomy compared with cirrhotic patients $(20.4 \%, n=22)(p<0.001)$. In addition, compared with patients who had cirrhosis, patients who did not have cirrhosis were more likely to have undergone a concomitant bile-duct resection (non-cirrhosis, $n=155$, $19.8 \%$ vs. cirrhosis, $n=3,2.8 \% ; p<0.001)$. Of note, while roughly half of patients without cirrhosis underwent a lymphadenectomy $(n=445,50.2 \%)$, less than 1 in 5 patients who had cirrhosis had any lymph nodes harvested at the time of surgery $(n=23,19.5 \%)(p<0.001)$. On pathological assessment, the median HLN was 3 (IQR, 1-6) among patients with cirrhosis compared with 4 (IQR, $2-8)$ among patients who did not have cirrhosis $(p=0.12)$. The median number of metastatic lymph nodes (MLN) was no different among patients who did $(n=1, \mathrm{IQR}, 1-3)$ and did not $(n=1$, IQR, $1-5)$ have cirrhosis $(p=0.51)$. On pathological assessment, there was also no difference in the proportion of patients who had R1 surgical margin (R1 margin: non-cirrhosis, $n=123,14.1 \%$ vs. cirrhosis, $n=10,8.5 \%)(p=0.11)$.

Following surgery, a total of 395 patients experienced a complication for an overall morbidity rate of $39.3 \%$. The incidence of any complication was slightly higher among patients who did not have cirrhosis $(n=360,40.6 \%)$ compared with patients who had cirrhosis $(n=35,29.7 \%)$ $(p=0.022)$. Particularly, the incidences of renal failure, pleural effusion, posthepatectomy liver failure (PHLF), hemorrhages, wound infections, respiratory, and cardiovascular complications were not different comparing noncirrhosis and cirrhosis groups (all $p>0.1$ ). In contrast, patients with cirrhosis group had a lower incidence of bile leakage and infections after surgery compared with noncirrhotic patients (bile leakage: non-cirrhosis, $n=59$, $6.6 \%$ vs. cirrhosis, $n=3,2.5 \%, p=0.08$; infections: noncirrhosis, $n=86,9.7 \%$ vs. cirrhosis, $n=4,3.4 \%$, $p=0.024$; Table 2). Interestingly, there was no difference
Table 1 Clinical and pathological features of the entire study cohort $(n=1005)$

\begin{tabular}{|c|c|c|c|}
\hline Variables & $\begin{array}{l}\text { Non-cirrhosis } \\
N(\%)\end{array}$ & $\begin{array}{l}\text { Cirrhosis } \\
N(\%)\end{array}$ & $p$ value \\
\hline Patients & $887(88.3 \%)$ & $118(11.7 \%)$ & - \\
\hline Age, median (IQR) & $\begin{array}{l}62 \text { years } \\
(53-70)\end{array}$ & $\begin{array}{l}56 \text { years } \\
\quad(46-65)\end{array}$ & $<0.001$ \\
\hline Gender & & & $<0.001$ \\
\hline Female & $420(47.4 \%)$ & $22(18.6 \%)$ & \\
\hline Male & $467(52.6 \%)$ & $96(81.4 \%)$ & \\
\hline ASA & & & 0.013 \\
\hline$\leq 2$ & $534(60.2 \%)$ & $85(72.0 \%)$ & \\
\hline$>2$ & $353(39.8 \%)$ & $33(28.0 \%)$ & \\
\hline $\begin{array}{l}\text { Neoadjuvant } \\
\text { chemotherapy }\end{array}$ & & & 0.14 \\
\hline No & $707(90.6 \%)$ & $97(95.1 \%)$ & \\
\hline Yes & $73(9.4 \%)$ & $5(4.9 \%)$ & \\
\hline Morphological type & & & 0.67 \\
\hline MF, IG & $677(83.1 \%)$ & $94(84.7 \%)$ & \\
\hline $\mathrm{PI}, \mathrm{MF}+\mathrm{PI}$ & $138(16.9 \%)$ & $17(15.3 \%)$ & \\
\hline Type of resections & & & $<0.001$ \\
\hline Wedge resection & $116(14.9 \%)$ & $35(32.4 \%)$ & \\
\hline Minor resection & $171(21.9 \%)$ & $51(47.2 \%)$ & \\
\hline Major resection & $494(63.2 \%)$ & $22(20.4 \%)$ & \\
\hline Margin status & & & 0.11 \\
\hline $\mathrm{R} 0$ & $749(85.9 \%)$ & $108(91.5 \%)$ & \\
\hline $\mathrm{R} 1$ & $123(14.1 \%)$ & $10(8.5 \%)$ & \\
\hline Lymph-node status & & & $<0.001$ \\
\hline Negative & $273(30.8 \%)$ & $11(9.3 \%)$ & \\
\hline Metastatic & $172(19.4 \%)$ & $12(10.2 \%)$ & \\
\hline Not harvested & $442(49.8 \%)$ & $95(80.5 \%)$ & \\
\hline Tumor size & & & $<0.001$ \\
\hline$\leq 5 \mathrm{~cm}$ & $345(38.9 \%)$ & $70(59.3 \%)$ & \\
\hline$>5 \mathrm{~cm}$ & $542(61.1 \%)$ & $48(40.7 \%)$ & \\
\hline Liver capsule involvement & & & 0.59 \\
\hline No & $758(85.5 \%)$ & $103(87.3 \%)$ & \\
\hline Yes & $129(14.5 \%)$ & $15(12.7 \%)$ & \\
\hline $\begin{array}{l}\text { Direct invasion adjacent } \\
\text { organs }\end{array}$ & & & 0.094 \\
\hline No & $748(95.8 \%)$ & $107(99.1 \%)$ & \\
\hline Yes & $33(4.2 \%)$ & $1(0.9 \%)$ & \\
\hline Major vascular resection & & & 0.083 \\
\hline No & $633(80.9 \%)$ & $94(87.9 \%)$ & \\
\hline Yes & $149(19.1 \%)$ & $13(12.2 \%)$ & \\
\hline Bile-duct resection & & & $<0.001$ \\
\hline No & $628(80.2 \%)$ & $105(97.2 \%)$ & \\
\hline Yes & $155(19.8 \%)$ & $3(2.8 \%)$ & \\
\hline Grade & & & 0.67 \\
\hline Well/moderate & $677(83.1 \%)$ & $94(84.7 \%)$ & \\
\hline Poorly/undifferentiated & $138(16.9 \%)$ & $17(15.3 \%)$ & \\
\hline Microvascular invasion & & & 0.041 \\
\hline No & $549(63.6 \%)$ & $85(73.3 \%)$ & \\
\hline
\end{tabular}


Table 1 continued

\begin{tabular}{llll}
\hline Variables & $\begin{array}{l}\text { Non-cirrhosis } \\
N(\%)\end{array}$ & $\begin{array}{l}\text { Cirrhosis } \\
N(\%)\end{array}$ & $p$ value \\
\hline Yes & $314(36.4 \%)$ & $31(26.7 \%)$ & \\
Perineural invasion & & & 0.002 \\
No & $570(74.7 \%)$ & $98(88.3 \%)$ & \\
Yes & $193(25.3 \%)$ & $13(11.7 \%)$ & \\
Multi-focal & & & 0.99 \\
No & $744(83.9 \%)$ & $99(83.9 \%)$ & \\
Yes & $143(16.1 \%)$ & $19(16.1 \%)$ & \\
Ca 19-9, median (IQR) & $52(17-232)$ & $31(17-65)$ & 0.18 \\
CEA, median (IQR) & $2.4(1.4-4.4)$ & $2.4(1.6-3.9)$ & 0.79 \\
Complication & & & 0.022 \\
No & $527(59.4 \%)$ & $83(70.3 \%)$ & \\
$\quad$ Yes & $360(40.6 \%)$ & $35(29.7 \%)$ & \\
Clavien-Dindo grade of & & & 0.48 \\
$\quad$ complication* & & & \\
1-2 & $204(56.7 \%)$ & $22(62.7 \%)$ & \\
$\geq 3$ & $156(43.3 \%)$ & $13(37.1 \%)$ & \\
Length of stay, median & $13(8-18)$ & $14(11-20)$ & 0.12 \\
$\quad$ IQR) & & & \\
Overall survival, 5 year & $40.9 \%$ & $39.0 \%$ & 0.19 \\
$\quad$ 95\% CI) & $(36.4-45.3)$ & $(28.7-49.2)$ & \\
\hline
\end{tabular}

$* N=395$ patients who had postoperative complications

$N A$ not available, $C I$ confidence interval

in the grade of complication (Clavien-Dindo grade $>3$ : non-cirrhosis, $n=156,43.3 \%$ vs. cirrhosis, $n=13$, $37.1 \%$ ) or length of stay (non-cirrhosis, 13 days vs. cirrhosis, 14 days) (both $p>0.1$ ).

Among the subset of patients who underwent a lymphadenectomy ( $n=468$. $46.6 \%$ ), the incidence of postoperative complications was comparable among patients who did (56.5\%) and did not $(42.9 \%)$ have cirrhosis $(p=0.21)$. In addition, among only patients who had cirrhosis, lymphadenectomy was associated with risk of complications (OR 4.31, 95\% 1.66-11.2) $(p=0.003)$. Among patients who did not have cirrhosis, there was an increased risk of infections among patients who underwent lymphadenectomy compared with patients who did not (infections: no lymphadenectomy, $n=26$, 5.9\% vs. lymphadenectomy, $n=60,13.5 \%) \quad(p<0.001)$; several specific complications were associated with lymphadenectomy when performed among cirrhotic patients (Table 3). Particularly, among patients with cirrhosis, lymphadenectomy was associated with higher risk of superficial wound infections (non-cirrhosis, $n=1,1.0 \%$ vs. cirrhosis, $n=2,8.7 \%, p=0.037$ ) and surgical site infections (non-cirrhosis, $n=0,0 \%$ vs. cirrhosis, $n=4$, $17.4 \%, p<0.001)$.
Table 2 Type of complications in non-cirrhosis and cirrhosis groups $(n=1005)$

\begin{tabular}{|c|c|c|c|}
\hline Variables & $\begin{array}{l}\text { Non- } \\
\text { cirrhosis } \\
N(\%)\end{array}$ & $\begin{array}{l}\text { Cirrhosis } \\
N(\%)\end{array}$ & $p$ value \\
\hline Patients & $887(88.3 \%)$ & $118(11.7 \%)$ & - \\
\hline Respiratory complications & & & 0.25 \\
\hline No & $848(95.6 \%)$ & $110(93.2 \%)$ & \\
\hline Yes & $39(4.4 \%)$ & $8(6.8 \%)$ & \\
\hline $\begin{array}{l}\text { Cardiovascular } \\
\text { complications }\end{array}$ & & & 0.56 \\
\hline No & $865(97.5 \%)$ & $114(96.6 \%)$ & \\
\hline Yes & $22(2.5 \%)$ & $4(3.4 \%)$ & \\
\hline Renal failure & & & 0.77 \\
\hline No & $875(98.7 \%)$ & $116(98.3 \%)$ & \\
\hline Yes & $12(1.3 \%)$ & $2(1.7 \%)$ & \\
\hline Pleura effusion & & & 0.79 \\
\hline No & $813(91.7 \%)$ & $109(92.4 \%)$ & \\
\hline Yes & $74(8.3 \%)$ & $9(7.6 \%)$ & \\
\hline $\begin{array}{l}\text { Posthepatectomy liver } \\
\text { failure }\end{array}$ & & & 0.75 \\
\hline No & $868(97.9 \%)$ & $116(98.3 \%)$ & \\
\hline Yes & $19(2.1 \%)$ & $2(1.7 \%)$ & \\
\hline Hemorrhage & & & 0.19 \\
\hline No & $867(97.8 \%)$ & $113(95.8 \%)$ & \\
\hline Yes & $20(2.2 \%)$ & $5(4.2 \%)$ & \\
\hline Bile leakage & & & 0.08 \\
\hline No & $828(93.4 \%)$ & $115(97.5 \%)$ & \\
\hline Yes & $59(6.6 \%)$ & $3(2.5 \%)$ & \\
\hline Wound infection & & & 0.97 \\
\hline No & $865(97.5 \%)$ & $115(97.5 \%)$ & \\
\hline Yes & $22(2.5 \%)$ & $3(2.5 \%)$ & \\
\hline Infections & & & 0.024 \\
\hline No & $801(90.3 \%)$ & $114(96.6 \%)$ & \\
\hline Yes & $86(9.7 \%)$ & $4(3.4 \%)$ & \\
\hline
\end{tabular}

\section{Propensity score matching analysis}

A propensity score matching was performed to minimize confounding and create more comparable cohorts $(n=150)$ among patients who did and did not have cirrhosis (Table 4). After propensity matching, demographic and clinicopathological characteristics among the cirrhotic and non-cirrhotic patients were comparable (Table 4). On propensity-matched analysis, the incidence of postoperative complications was $32.0 \%(n=24)$ in both cohorts of patients $(p>0.99$; Table 4$)$. Interestingly, among the 24 patients who did not have cirrhosis yet had a complication, 1 (4.2\% of 24) patient had a severe complication (ClavienDindo grade of complication $\geq 3$ ) versus 5 (20.8\% of 24 ) patients in the cirrhosis group $(p=0.08)$. Among patients who did not have cirrhosis, the incidence of complications 
Table 3 Type of complications in non-cirrhosis and cirrhosis groups for patients who underwent and did not undergo lymphadenectomy $(n=1005)$

\begin{tabular}{|c|c|c|c|c|c|c|}
\hline & \multicolumn{2}{|l|}{$\begin{array}{l}\text { Non-cirrhosis } \\
N(\%)\end{array}$} & \multirow[t]{2}{*}{$p$ value } & \multicolumn{2}{|l|}{$\begin{array}{l}\text { Cirrhosis } \\
N(\%)\end{array}$} & \multirow[t]{2}{*}{$p$ value } \\
\hline & No Lymphad. & Lymphad & & No Lymphad. & Lymphad & \\
\hline Patients & $442(49.8 \%)$ & $445(50.2 \%)$ & - & $95(80.5 \%)$ & $23(19.5 \%)$ & - \\
\hline Respiratory complications & & & 0.64 & & & $<0.00$ \\
\hline No & $424(95.9 \%)$ & $424(95.3 \%)$ & & $93(97.9 \%)$ & $17(73.9 \%)$ & 1 \\
\hline Yes & $18(4.1 \%)$ & $21(4.7 \%)$ & & $2(2.1 \%)$ & $6(26.1 \%)$ & \\
\hline Cardiovascular complications & & & 0.68 & & & 0.00 \\
\hline No & $432(97.7 \%)$ & $433(97.3 \%)$ & & $94(99.0 \%)$ & $20(86.9 \%)$ & 4 \\
\hline Yes & $10(2.3 \%)$ & $12(2.7 \%)$ & & $1(1.0 \%)$ & $3(13.0 \%)$ & \\
\hline Renal failure & & & 0.57 & & & 0.48 \\
\hline No & $437(98.9 \%)$ & $438(98.4 \%)$ & & $93(97.9 \%)$ & $23(100 \%)$ & \\
\hline Yes & $5(1.1 \%)$ & $7(1.6 \%)$ & & $2(2.1 \%)$ & - & \\
\hline \multicolumn{7}{|l|}{ Pleura effusion } \\
\hline No & $387(87.6 \%)$ & $426(95.7 \%)$ & & $89(93.7 \%)$ & $20(86.9 \%)$ & \\
\hline Yes & $55(12.4 \%)$ & $19(4.3 \%)$ & $<0.001$ & $6(6.3 \%)$ & $3(13.0 \%)$ & 0.28 \\
\hline Posthepatectomy liver failure & & & 0.81 & & & 0.00 \\
\hline No & $432(97.7 \%)$ & $436(98.0 \%)$ & & $95(100 \%)$ & $21(91.3 \%)$ & 4 \\
\hline Yes & $10(2.3 \%)$ & $9(2.0 \%)$ & & - & $2(8.7 \%)$ & \\
\hline Hemorrhage & & & 0.66 & & & 0.24 \\
\hline No & $433(98.0 \%)$ & $434(97.5 \%)$ & & $92(96.8 \%)$ & $21(91.3 \%)$ & \\
\hline Yes & $9(2.0 \%)$ & $11(2.5 \%)$ & & $3(3.2 \%)$ & $2(8.7 \%)$ & \\
\hline Bile leakage & & & 0.24 & & & 0.037 \\
\hline No & $417(94.3 \%)$ & $411(92.4 \%)$ & & $94(99.0 \%)$ & $21(91.3 \%)$ & \\
\hline Yes & $25(5.7 \%)$ & $34(7.6 \%)$ & & $1(1.0 \%)$ & $2(8.7 \%)$ & \\
\hline Wound infection & & & 0.99 & & & 0.037 \\
\hline No & $431(97.5 \%)$ & $434(97.5 \%)$ & & $94(99.0 \%)$ & $21(91.3 \%)$ & \\
\hline Yes & $11(2.5 \%)$ & $11(2.5 \%)$ & & $1(1.0 \%)$ & $2(8.7 \%)$ & \\
\hline Infections & & & $<0.00$ & & & $<0.00$ \\
\hline No & $416(94.1 \%)$ & $385(86.5 \%)$ & 1 & $95(100 \%)$ & $19(82.6 \%)$ & 1 \\
\hline Yes & $26(5.9 \%)$ & $60(13.5 \%)$ & & - & $4(17.4 \%)$ & \\
\hline
\end{tabular}

was $42.9 \%(N=6)$ among patients who underwent lymphadenectomy versus $29.5 \%(N=18)$ among patients who did not undergo lymphadenectomy ( $p=0.33$; Fig. 1a). In contrast, the incidence of complication was $71.4 \%$ $(N=10)$ among patients who underwent lymphadenectomy versus $22.9 \%(N=14)$ among patients who did not undergo lymphadenectomy (OR 8.39, 95\% CI 2.3-30.9) ( $p<0.001$; Fig. 1b). In the propensity-matched analysis, the median HLN was comparable among patients independent of cirrhosis status ( $p=0.95$; Fig. $2 \mathrm{a}$ ) and was not associated with the occurrence of complications ( $p=0.68$; Fig. 2b). While lymphadenectomy was associated with a higher risk of infections (non-cirrhosis, $n=0,0 \%$ vs. cirrhosis, $n=3,21.4 \%, p<0.001$ ) among patients with cirrhosis, infections were not associated with lymphadenectomy among patients who did not have cirrhosis $(p=0.19)$ (Supplementary Tables $1 \mathrm{~S}$ and $2 \mathrm{~S}$ ).

\section{Discussion}

Cirrhosis can be a common finding among patients with liver malignancies. While the association of cirrhosis with HCC is well established [19], more recent data have established that cirrhosis can also significantly increase the risk of ICC [8-10]. In turn, cirrhosis can have important implications in the peri-operative management of patients undergoing liver surgery increasing the risk of morbidity and mortality associated with liver resection [17]. Since lymphadenectomy is not commonly performed for HCC, the potential incremental risk of lymph-node dissection in 
Table 4 Clinical and pathological features of patients after propensity score matching analysis $(n=150)$

\begin{tabular}{|c|c|c|c|}
\hline Variables & $\begin{array}{l}\text { Non-cirrhosis } \\
N(\%)\end{array}$ & $\begin{array}{l}\text { Cirrhosis } \\
N(\%)\end{array}$ & $p$ value \\
\hline Patients & $75(50.0 \%)$ & $75(50.0 \%)$ & - \\
\hline Age, median (IQR) & 57 years $(48-64)$ & 54 years $(46-66)$ & 0.72 \\
\hline Gender & & & 0.57 \\
\hline Female & $20(26.7 \%)$ & $17(22.7 \%)$ & \\
\hline Male & $55(73.3 \%)$ & $58(77.3 \%)$ & \\
\hline ASA & & & 0.55 \\
\hline$\leq 2$ & $61(81.3 \%)$ & $58(77.3 \%)$ & \\
\hline$>2$ & $14(18.7 \%)$ & $17(22.7 \%)$ & \\
\hline Neoadjuvant chemotherapy & & & 0.30 \\
\hline No & $69(98.6 \%)$ & $66(95.6 \%)$ & \\
\hline Yes & $1(1.4 \%)$ & $3(4.4 \%)$ & \\
\hline Morphological type & & & 0.98 \\
\hline MF, IG & $70(95.9 \%)$ & $71(95.9 \%)$ & \\
\hline $\mathrm{PI}, \mathrm{MF}+\mathrm{PI}$ & $3(4.1 \%)$ & $3(4.1 \%)$ & \\
\hline Type of resections & & & 0.97 \\
\hline Wedge resection & $23(32.4 \%)$ & $23(32.4 \%)$ & \\
\hline Minor resection & $32(45.1 \%)$ & $33(46.5 \%)$ & \\
\hline Major resection & $16(22.5 \%)$ & $15(21.1 \%)$ & \\
\hline Margin status & & & 0.47 \\
\hline R0 & $70(93.3 \%)$ & $72(96.0 \%)$ & \\
\hline $\mathrm{R} 1$ & $5(6.7 \%)$ & $3(4.0 \%)$ & \\
\hline Lymph-node status & & & 0.93 \\
\hline Negative & $9(12.0 \%)$ & $8(10.7 \%)$ & \\
\hline Metastatic & $5(6.7 \%)$ & $6(8.0 \%)$ & \\
\hline Not harvested & $61(81.3 \%)$ & $61(81.3 \%)$ & \\
\hline Tumor size & & & 0.86 \\
\hline$\leq 5 \mathrm{~cm}$ & $51(68.0 \%)$ & $50(66.7 \%)$ & \\
\hline$>5 \mathrm{~cm}$ & $24(32.0 \%)$ & $25(33.3 \%)$ & \\
\hline Liver capsule involvement & & & 0.55 \\
\hline No & $68(90.7 \%)$ & $70(93.3 \%)$ & \\
\hline Yes & $7(9.3 \%)$ & $5(6.7 \%)$ & \\
\hline Direct invasion adjacent organs & & & $>0.99$ \\
\hline No & $70(98.6 \%)$ & $70(98.6 \%)$ & \\
\hline Yes & $1(1.4 \%)$ & $1(1.4 \%)$ & \\
\hline Major vascular resection & & & 0.31 \\
\hline No & $69(92.0 \%)$ & $71(95.9 \%)$ & \\
\hline Yes & $6(8.0 \%)$ & $3(4.1 \%)$ & \\
\hline Bile-duct resection & & & 0.56 \\
\hline No & $69(97.2 \%)$ & $70(98.6 \%)$ & \\
\hline Yes & $2(2.8 \%)$ & $1(1.4 \%)$ & \\
\hline Grade & & & 0.51 \\
\hline Well/moderate & $67(93.1 \%)$ & $63(90.0 \%)$ & \\
\hline Poorly/undifferentiated & $5(6.9 \%)$ & $7(10.0 \%)$ & \\
\hline Microvascular invasion & & & 0.82 \\
\hline No & $62(83.8 \%)$ & $63(85.1 \%)$ & \\
\hline Yes & $12(16.2 \%)$ & $11(14.9 \%)$ & \\
\hline Perineural invasion & & & 0.15 \\
\hline No & $63(87.5 \%)$ & $68(94.4 \%)$ & \\
\hline
\end{tabular}


Table 4 continued

\begin{tabular}{lll}
\hline Variables & $\begin{array}{l}\text { Non-cirrhosis } \\
N(\%)\end{array}$ & $\begin{array}{l}\text { Cirrhosis } \\
N(\%)\end{array}$ \\
\hline Yes & $9(12.5 \%)$ & $4(5.6 \%)$ \\
Multi-focal & & $69(92.0 \%)$ \\
No & $67(89.3 \%)$ & $6(8.0 \%)$ \\
Yes & $8(10.7 \%)$ & $33(17-66)$ \\
Ca 19-9, median (IQR) & $30(12-77)$ & $2.3(1.6-3.7)$ \\
CEA, median (IQR) & $2.4(1.6-3.4)$ & \\
Complication & & $51(68.0 \%)$ \\
No & $51(68.0 \%)$ & $24(32.0 \%)$ \\
Yes & $24(32.0 \%)$ & $19(79.2 \%)$ \\
Clavien-Dindo grade of complication* & & $5(20.8 \%)$ \\
$1-2$ & $23(95.8 \%)$ & $14(11-20)$ \\
$\geq 3$ & $1(4.2 \%)$ & $48.7 \%(35.8-60.5)$ \\
Length of stay, median (IQR) & $14(10-17)$ & 0.27 \\
Overall survival, 5 year (95\% CI) & $50.0 \%(35.0-63.3)$ & 0.99 \\
\hline
\end{tabular}

$* N=48$ patients who had postoperative complications

$N A$ not available, $C I$ confidence interval

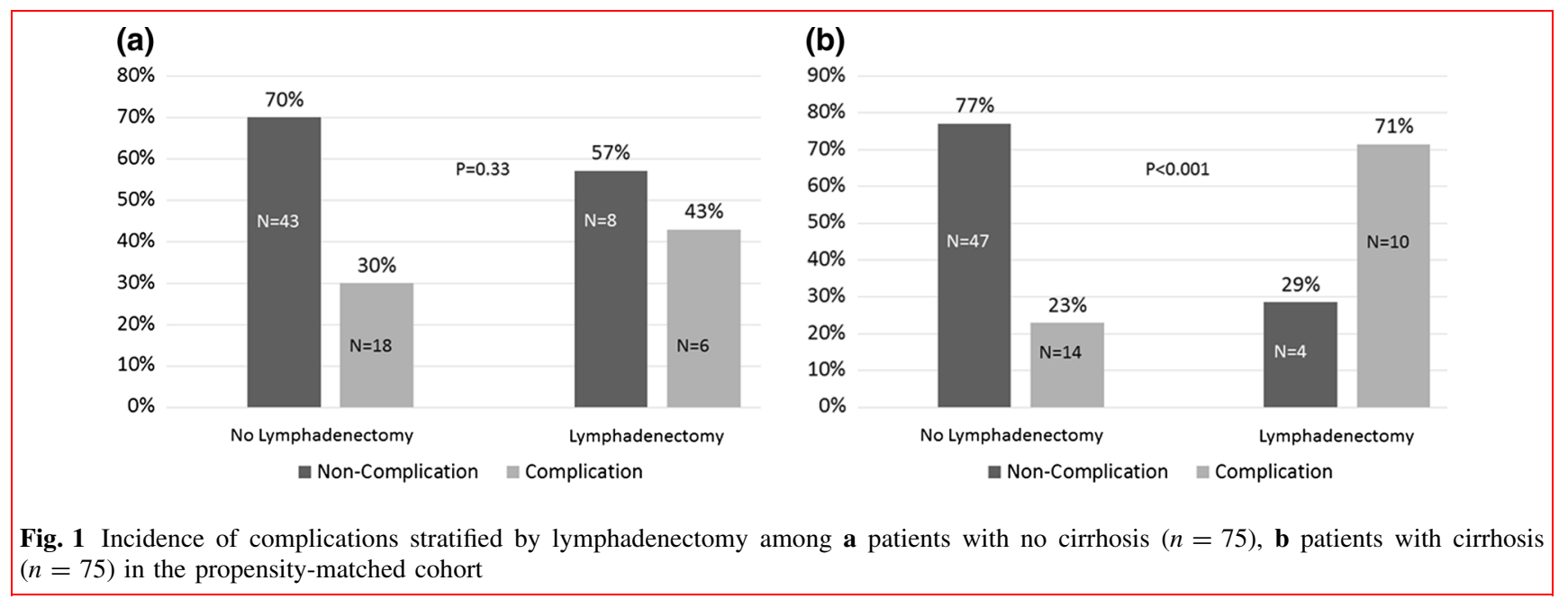

the setting of cirrhosis and HCC has not been clinically relevant. The topic of lymphadenectomy is much more relevant among ICC patients as the 8th edition of the AJCC staging system now recommends that surgeons perform a lymphadenectomy [13]. The current study was important because it specifically examined peri-operative outcomes of that subset of ICC patients who had concomitant cirrhosis. While the incidence of any complication was not higher among patients who had cirrhosis, this group of patients was more likely to undergo a minor versus major hepatectomy. Interestingly, while roughly half of patients without cirrhosis underwent a lymphadenectomy, only about $20 \%$ of patients with cirrhosis had any LNH at the time of surgery. When lymph nodes were indeed evaluated, however, the incidence of MLN was comparable among patients who did and did not have cirrhosis. On propensity score matching analysis, lymphadenectomy was associated with an increased risk of morbidity among patients with cirrhosis compared with patients who did not have cirrhosis. To our knowledge, these data are the first to examine specifically the topic of lymphadenectomy for ICC among patients with cirrhosis.

A recent population-based study using the National Health and Nutrition Survey data estimated that the prevalence of cirrhosis was approximately $0.27 \%$ in the USA [20]. The authors also noted that male sex and older age were independently associated with the risk of cirrhosis [20]. In the current study, we noted that the incidence of 

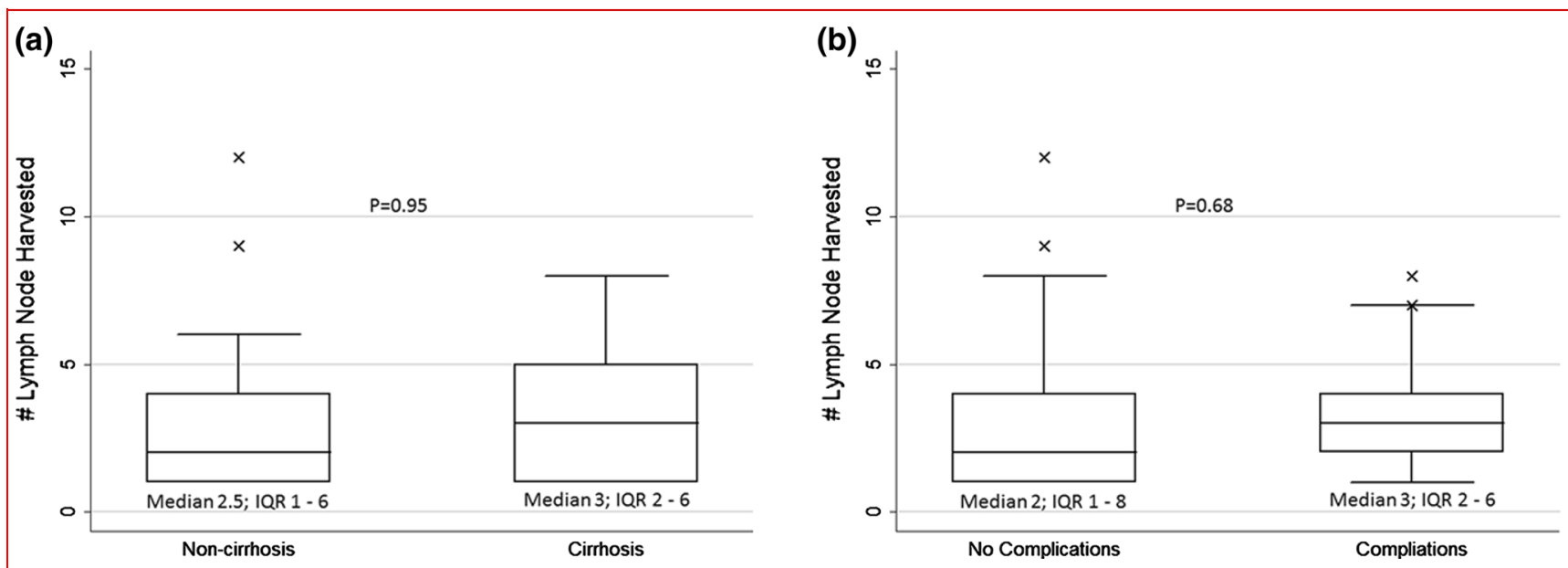

Fig. 2 a Number of lymph node harvested among patients with no cirrhosis $(n=75)$ versus patients with cirrhosis $(n=75)$ in the propensitymatched cohort. b Number of lymph node harvested among patients who had no complications $(n=102)$ versus patients who experience at least one complication $(n=48)$

cirrhosis among ICC patients was higher among men, yet the median age of patients with ICC and cirrhosis was younger than non-cirrhotic patients. Cirrhosis is an important risk factor for ICC, as several studies have reported a strong association between cirrhosis and ICC [21-23]. Several studies from Eastern countries have noted an incidence of cirrhosis as high as 20-25\% among patients with ICC [24, 25]. Conversely, in most Western series, the incidence of cirrhosis has been somewhat lower at about $10 \%$ [26-28]. In the current study, roughly 1 in 10 patients who had ICC also had pathological evidence of underlying cirrhosis. Differences in the reported incidences of cirrhosis are undoubtedly multi-factorial and may be related to regional epidemiological differences in terms of incidence of environmental risk factors (e.g., hepatitis, obesity, and hepatolithiasis). The "intermediate" incidence of cirrhosis reported in the current study likely reflects the multinational character of our database. In fact, in our study cohort, the incidence of cirrhotic patients was $2.9 \%$ among centers in the USA versus $9.5 \%$ among European centers and $19.2 \%$ among Eastern centers $(p<0.001)$.

Perhaps not surprisingly, there were several differences in the baseline clinicopathological and surgical characteristics among cirrhotic versus non-cirrhotic patients. For example, the size of ICC tumors was smaller among patients with cirrhosis. Patients who had cirrhosis underwent fewer major hepatectomies, as well as were less likely to have a concomitant major vascular $(\Delta=-7 \%)$ or bileduct $(\Delta=-17 \%)$ resection. In addition, patients with cirrhosis were also less likely to undergo lymphadenectomy $(\Delta=-30 \%)$. Previous data from our own group had noted that utilization of lymphadenectomy among patients with ICC varied considerably [29-31]. In fact, the odds of performing a lymphadenectomy has increase over time as more and more surgeons have accepted the importance of nodal staging for ICC [32]. Furthermore, surgeons are more prone to perform a lymphadenectomy among patients with more advanced T-stage disease [32]. The current study expands on this previous work as it noted that the presence of cirrhosis also significantly influenced the surgical decision to perform a lymphadenectomy. In fact, patients with cirrhosis were about a third less likely to have a lymph node evaluated. These data are important especially in light of the finding that when lymphadenectomy was performed, the chance of finding metastatic disease in the nodal basin was comparable among cirrhotic and non-cirrhotic patients.

Given the varied baseline clinical and surgical details among cirrhotic and non-cirrhotic patients, a propensity score match analysis was performed to obtain a more comparable cohort of patients. Among the 150 propensitymatched patients, the overall incidence of morbidity was comparable among cirrhotic and non-cirrhotic patients $(\approx 30 \%)$. Of note, the incidence of severe complication (Clavien-Dindo grade $\geq 3$ ) was, however, fivefold higher among patients with cirrhosis compared with non-cirrhotic patients (non-cirrhosis $4.2 \%$ vs. cirrhosis 20.8\%). Interestingly, among non-cirrhotic patients, there was no difference in the risk of morbidity among patients with or without lymphadenectomy. In contrast, among patients who had cirrhosis, the risk of a complication was roughly threefold higher among patients who had a lymphadenectomy $(71 \%)$ compared with patients who did not have a lymphadenectomy $(23 \%) \quad(p<0.001)$. These data are consistent with findings from previous studies that have evaluated lymphadenectomy and cirrhosis for other malignancies [14-16]. In one study that examined patients with gastric cancer, the authors reported that lymphadenectomy in the presence of cirrhosis was associated 
with a great risk of complications including infection and ascites [15]. Interestingly, in the current study we similarly noted that lymphadenectomy in the setting of cirrhosis was associated with a higher risk of infection, yet not ascites. As such, the data would collectively suggest that the benefit of lymphadenectomy in cirrhotic patients should be considered relative to the somewhat higher morbidity.

Several limitations should be considered when interpreting the results. Given the retrospective design and the multicenter nature of the study, selection bias was possible. Undoubtedly, there were some patients with cirrhosis who were not offered surgery due to the poor underlying quality of their non-tumorous hepatic parenchyma. In addition, only Child-Pugh Class A patients were included in the current study and therefore the results should not be extrapolated to patients with more advanced cirrhosis. The study cohort also included patients who underwent liver surgery at one of fourteen hepatobiliary centers in the USA, Europe, Australia, and Asia. The results from the current study may therefore not be generalizable to non-academic, community centers or lower volume hepatobiliary centers.

In conclusion, the incidence of cirrhosis among ICC patients undergoing surgical management was $12 \%$. The incidence of cirrhosis varied somewhat by center and geographic region. Patients who had cirrhosis were less likely to undergo a major hepatectomy and were about onethird less likely to undergo lymphadenectomy at the time of surgery. The incidence of MLN was comparable among cirrhotic and non-cirrhotic patients when lymph-node evaluation was performed. On propensity matching, even after controlling for competing risk factors, patients with cirrhosis had a higher likelihood of complications when a lymphadenectomy was performed. As such, the AJCC 8th edition recommendation to perform an extended lymphnode harvest should be considered in light of these data when operating on patients with ICC and cirrhosis.

\section{References}

1. Plentz RR, Malek NP (2015) Clinical presentation, risk factors and staging systems of cholangiocarcinoma. Best Pract Res Clin Gastroenterol 29:245-252

2. Shin HR, Oh JK, Masuyer E et al (2010) Epidemiology of cholangiocarcinoma: an update focusing on risk factors. Cancer Sci 101:579-585

3. Chan-On W, Kuwahara K, Kobayashi N et al (2009) Cholangiocarcinomas associated with long-term inflammation express the activation-induced cytidine deaminase and germinal centerassociated nuclear protein involved in immunoglobulin V-region diversification. Int J Oncol 35:287-295

4. Goral V (2017) Cholangiocarcinoma: new insights. Asian Pac J Cancer Prev 18:1469-1473

5. Thinkhamrop K, Khuntikeo N, Phonjitt P et al (2015) Association between diabetes mellitus and fatty liver based on ultrasonography screening in the world's highest cholangiocarcinoma incidence region, Northeast Thailand. Asian Pac J Cancer Prev 16:3931-3936

6. Zhang H, Yang T, Wu M et al (2016) Intrahepatic cholangiocarcinoma: epidemiology, risk factors, diagnosis and surgical management. Cancer Lett 379:198-205

7. Welzel TM, Graubard BI, El-Serag HB et al (2007) Risk factors for intrahepatic and extrahepatic cholangiocarcinoma in the United States: a population-based case-control study. Clin Gastroenterol Hepatol 5:1221-1228

8. Shaib YH, Davila JA, McGlynn K et al (2004) Rising incidence of intrahepatic cholangiocarcinoma in the United States: a true increase? J Hepatol 40:472-477

9. Peng NF, Li LQ, Qin X et al (2011) Evaluation of risk factors and clinicopathologic features for intrahepatic cholangiocarcinoma in Southern China: a possible role of hepatitis B virus. Ann Surg Oncol 18:1258-1266

10. Michelotti GA, Machado MV, Diehl AM (2013) NAFLD NASH and liver cancer. Nat Rev Gastroenterol Hepatol 10:656-665

11. Cauchy F, Fuks D, Zarzavadjian Le Bian A et al (2014) Metabolic syndrome and non-alcoholic fatty liver disease in liver surgery: the new scourges? World J Hepatol 6:306-314

12. Shaib YH, El-Serag HB, Davila JA et al (2005) Risk factors of intrahepatic cholangiocarcinoma in the United States: a casecontrol study. Gastroenterology 128:620-626

13. Amin MB, American Joint Committee on Cancer (2017) AJCC cancer staging manual Chicago, American Joint Committee on Cancer, Springer

14. Bagante F, Spolverato G, Weiss M et al. (2018) Assessment of the lymph node status in patients undergoing liver resection for intrahepatic cholangiocarcinoma: the new eighth edition AJCC staging system. J Gastrointest Surg 22(1):52-59

15. Lee JH, Kim J, Cheong JH et al (2005) Gastric cancer surgery in cirrhotic patients: result of gastrectomy with D2 lymph node dissection. World J Gastroenterol 11:4623-4627

16. Tachibana M, Kotoh T, Kinugasa S et al (2000) Esophageal cancer with cirrhosis of the liver: results of esophagectomy in 18 consecutive patients. Ann Surg Oncol 7:758-763

17. Hackl C, Schlitt HJ, Renner P et al (2016) Liver surgery in cirrhosis and portal hypertension. World J Gastroenterol 22:2725-2735

18. Austin PC, Grootendorst P, Anderson GM (2007) A comparison of the ability of different propensity score models to balance measured variables between treated and untreated subjects: a Monte Carlo study. Stat Med 26:734-753

19. El-Serag HB (2011) Hepatocellular carcinoma. N Engl J Med 365:1118-1127

20. Scaglione S, Kliethermes S, Cao G et al (2015) The epidemiology of cirrhosis in the United States: a population-based study. J Clin Gastroenterol 49:690-696

21. Affo S, Yu LX, Schwabe RF (2017) The role of cancer-associated fibroblasts and fibrosis in liver cancer. Annu Rev Pathol 12:153-186

22. Vijgen S, Terris B, Rubbia-Brandt L (2017) Pathology of intrahepatic cholangiocarcinoma. Hepatobiliary Surg Nutr 6:22-34

23. Tyson GL, El-Serag HB (2011) Risk factors for cholangiocarcinoma. Hepatology 54:173-184

24. Jeong S, Cheng Q, Huang L et al (2017) Risk stratification system to predict recurrence of intrahepatic cholangiocarcinoma after hepatic resection. BMC Cancer 17:464

25. Wang Y, Li J, Xia Y et al (2013) Prognostic nomogram for intrahepatic cholangiocarcinoma after partial hepatectomy. J Clin Oncol 31:1188-1195

26. Ali SM, Clark CJ, Mounajjed T et al (2015) Model to predict survival after surgical resection of intrahepatic cholangiocarcinoma: the Mayo Clinic experience. HPB (Oxford) 17:244-250 
27. Doussot A, Lim C, Gomez Gavara C et al (2016) Multicentre study of the impact of morbidity on long-term survival following hepatectomy for intrahepatic cholangiocarcinoma. Br J Surg 103:1887-1894

28. Doussot A, Gonen M, Wiggers JK et al (2016) Recurrence patterns and disease-free survival after resection of intrahepatic cholangiocarcinoma: preoperative and postoperative prognostic models. J Am Coll Surg 223:493-505

29. Kim Y, Moris DP, Zhang XF et al. (2017) Evaluation of the 8th edition American Joint Commission on Cancer (AJCC) staging system for patients with intrahepatic cholangiocarcinoma: a surveillance, epidemiology, and end results (SEER) analysis. J Surg Oncol 116(6):643-650

30. Bagante F, Gani F, Spolverato G et al (2015) Intrahepatic cholangiocarcinoma: prognosis of patients who did not undergo lymphadenectomy. J Am Coll Surg 221:e1031-e1034

31. Kim Y, Spolverato G, Amini N et al (2015) Surgical management of intrahepatic cholangiocarcinoma: defining an optimal prognostic lymph node stratification schema. Ann Surg Oncol 22:2772-2778

32. Zhang XF, Chakedis J, Bagante $F$ et al (accepted) Trends in utilization of lymphadenectomy in curative-intent surgery for intrahepatic cholangiocarcinoma. Brit J Surg 